\title{
SIMPLE EVAPORATION CONTROLLER FOR THIN FILM DEPOSITION FROM A RESISTIVELY HEATED BOAT
}

\author{
John H. Scofield, Lou Bajuk, and William Mohler \\ Department of Physics \\ Oberlin College \\ Oberlin, $\mathrm{OH} 44074$
}

\begin{abstract}
A simple, inexpensive circuit is described for switching the current through a resistively heated evaporation boat during thin film deposition. The circuit uses an SCR to switch the $0-15 \mathrm{~A}$ current in the primary of a $2 \mathrm{KVA}$ step-down transformer that supplies the 0-200A current to an evaporation boat. The circuit is controlled by a 0-10VDC signal similar to that furnished by an Inficon XTC deposition rate controller. This circuit may be assembled from a handful of parts for a cost of about $\$ 400$, nearly $1 / 10$ th the cost of similar commercial units. Minimum construction is required, since the circuit is built around an off-the-shelf, self-contained SCR unit.
\end{abstract}

\section{Introduction}

A common method for fabricating thin coatings of an element (or compound) is to evaporate a solid charge of the element from a resistively heated tungsten "boat," allowing the vapor to collect onto the substrate used to support the thin film [1]. The low-voltage, high-current used to heat the tungsten boat is typically supplied by a the secondary of a 2 $\mathrm{kVA}$, step-down transformer. In the simplest arrangement, the deposition rate is controlled manually by adjusting the voltage across the primary of the filament transformer with a Variac. If the charge is evaporated to completion, the final film thickness is determined by the mass of the charge and various geometry factors. To add the above apparatus to an existing vacuum system requires a filament transformer, Variac, high-current vacuum feed-throughs, and an evaporation boat. The required items may be purchased commercially from a vacuum equipment vendor (for instance, from Veeco) for an amount ranging from $2-3 \mathrm{~K} \$$. The transformer may be obtained with substantial savings from an alternate supplier.

Without further instrumentation it is difficult to control the deposition rate. Improved control is obtained by using a deposition rate monitor. While a variety of methods are available, the simplest and most popular is to use a quartz crystal monitor. For this type of monitor, the quartz crystal of an oscillator is mounted in the vacuum chamber so that the evaporated material collects on its surface during deposition. By monitoring the oscillator frequency during deposition, the mass of the deposited film may be determined as a function of time, from which the deposition rate is determined. Since thermal time constants are long, a person may maintain a fairly steady rate by simply adjusting the Variac during film deposition to maintain the desired rate as displayed on the deposition rate monitor. The sensor, vacuum feed- thru, and electronics for such a monitor can cost from 2-4 k\$. 
In many cases, manual deposition control is not sufficient so that an electronic feedback control system is required. Such is the case, for instance, in depositing alloys from two or more sources operated simultaneously. An error signal supplied by the deposition rate monitor/controller must be fed back to control the current to the evaporation boat. Many of the commercially available deposition rate monitors (i.e., controllers) have built-in Proportional-Integral-Differential (PID) circuitry for feedback control. They are unable, however, to directly switch the high current of the transformer. Instead, they typically furnish a $0-10 \mathrm{~V}$ control signal that may be used to control an external high-current, switching circuit. Manufacturers like Veeco, for instance, sell an "evaporation controller" to perform the high-current switching. This unit, built around a silicon controlled rectifier (SCR), costs about $3 \mathrm{~K} \$$. Here we describe an alternative that is easy to construct, and costs about $\$ 400$.

\section{Inexpensive High-Current Switch}

Our solution is built around an inexpensive, commercially-available SCR module, the Eurotherm Model 831. This SCR unit sells for about $\$ 350$, and, as purchased, is well suited for this application. A minor difficulty arises owing to the fact that the model 831 requires a 4-20mA control signal, whereas our deposition rate controller (an Inficon model XTC) supplies a 0-10V signal. The problem is easily overcome by inserting a voltage-to-current converter circuit between the two. The scheme is shown in Figure 1. It is not necessary to add an ammeter since the Eurotherm model 831 SCR unit includes an LED current meter on its front panel. The SCR unit also comes with a built in fuse and adjustments for maximum allowed current.

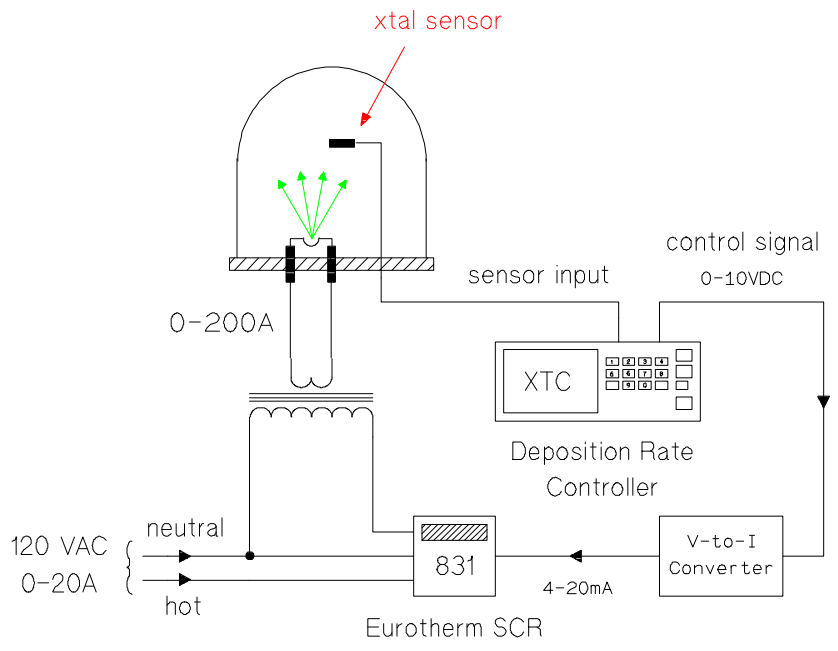

Figure 1. Block diagram for the evaporation feedback control circuit.

\section{Voltage-to-Current Converter}

The voltage-to-current converter circuit, shown in Figure 2, is similar to one found elsewhere [2]. The circuit, built around a unipolar op-amp, is powered from a floating 9V DC power supply [3]. The maximum current drain on the $9 \mathrm{~V}$ supply is $45 \mathrm{~mA}$. 


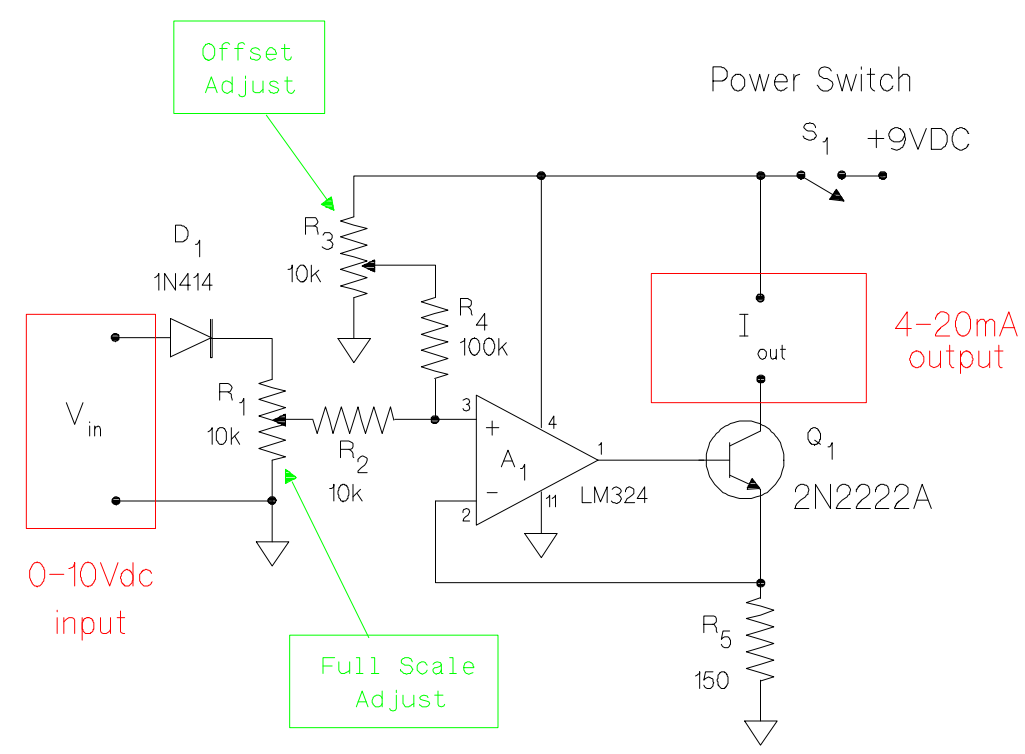

Figure 2. Schematic diagram for the voltage-to-current converter circuit. The circuit is designed to take the $0-10$ VDC control voltage supplied by the deposition rate controller, and furnish the 4-20 mA control current to the model 831 SCR module.

With $\mathrm{S}_{1}$ set to auto, the circuit works as follows. The 0-10VDC input signal from the deposition rate controller is attenuated by $\mathrm{R}_{1}$ (adjustable) to a value between 09.3VDC. Diode $\mathrm{D}_{1}$ protects the unit from input voltages of the wrong polarity. The attenuated control voltage $\left(\mathrm{E}_{+}\right)$is then fed into the non-inverting op-amp input. The fixed resistor $\mathrm{R}_{5}$ is the current-sensing resistor, providing a voltage feedback signal ( $\left.\mathrm{E}_{-}\right)$to the inverting input of the op-amp. The op-amp adjusts the base current of $\mathrm{Q}_{1}$ to maintain $\mathrm{E}_{+}$ $=\mathrm{E}_{-}$. Given a fixed value for $\mathrm{E}_{+}$, the circuit will maintain a constant current through $\mathrm{R}_{2}$, the collector of $\mathrm{Q}_{1}$, and hence the Eurotherm SCR unit. The value of $\mathrm{R}_{5}$ was chosen so that a maximum of $22 \mathrm{~mA}$ flows through it and the $260 \mathrm{Ohm}$ internal resistance of the SCR module when the transistor is fully turned on. Trimmer pot $\mathrm{R}_{3}$ is used to provide an offset, so that $4 \mathrm{~mA}$ output current is delivered to the SCR unit when zero input voltage is supplied. Fixed resistors $\mathrm{R}_{2}$ and $\mathrm{R}_{4}$ act as a summing network for the input control voltage and the DC offset. The voltage-to-current circuit is calibrated by adjusting trim pot $\mathrm{R}_{3}$ for $4 \mathrm{~mA}$ out with $0 \mathrm{VDC}$ in and adjusting trimmer pot $\mathrm{R}_{1}$ for $20 \mathrm{~mA}$ out with 10 VDC in.

There are a variety of ways to add manual evaporation control to the circuit. We decided to add manual evaporation control by providing an alternate 4-20 mA control current to the SCR unit from a rheostat (see Figure 2). With switch $\mathrm{S}_{1}$ set to manual, resistors $\mathrm{R}_{6}$ and $\mathrm{R}_{7}$ combine with the $260 \mathrm{Ohm}$ internal resistance of the model 831 to provide the 4-20 mA manual control from the 9VDC supply. Manual evaporation control remains available should failure occur in the V-to-I converter circuit.

In conclusion, we have presented an inexpensive circuit for switching the high current of a filament transformer during thermal evaporation. The circuit may be used along with a deposition rate controller to perform thermal evaporation under feedback control. 
Scofield, et. al., Review of Scientific Instruments 61, 1994-6 (July 1990)

\section{Acknowledgments}

This work was supported by the National Aeronautics and Space Administration Lewis Research Center, Grant No. NAG 3-885.

\section{References}

1. Leon I. Maissel and Reinhard Glang, editors, Handbook of Thin Film Technology (McGraw-Hill Book Co., New York, 1970).

2. Walter G. Jung, IC Op-Amp Cookbook, 3rd edition (Howard W. Sams \& Co., Indianapolis, 1987).

3. The $9 \mathrm{~V}$ dc supply must be floating (i.e., not have either side grounded) since the Eurotherm model 831 input cannot be referenced to ground. The Inficon XTC deposition controller is already floating. 